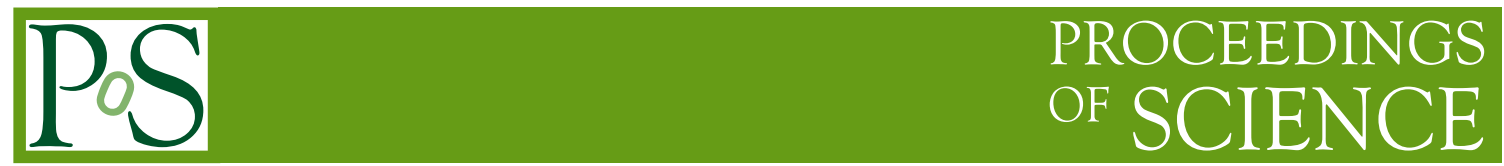

\title{
A systematic search for periodicities of X-ray binaries in the Swift BAT data
}

\author{
Rosa Poggiani* \\ Università di Pisa \\ E-mail: rosa.poggianiedf.unipi.it
}

\begin{abstract}
I present the results of a systematic search for long term periodicities of the X-ray binaries observed with Swift Burst Alert Monitor (BAT) during the first ten years of operation. The instrument monitors more than $50 \%$ of the sky each day, providing long term light curves of several hundreds sources. The detection of periodicities in the light curves of X-ray binaries is a tool to understand the geometry and the emission properties of these systems. The search has been performed using the SparSpec algorithm. The periodicities detected in the Swift BAT data are compared with those detected in the RXTE ASM data with the same method. Several orbital and superorbital periodicities previously reported in literature have been detected.
\end{abstract}

Swift: 10 Years of Discovery,

2-5 December 2014

La Sapienza University, Rome, Italy

${ }^{*}$ Speaker. 


\section{Introduction}

Periodic variations, with periods of a few days or longer, have been observed in the light curves of several Low Mass X-ray and High Mass X-ray binaries [四]. The orbital motion is a natural candidate for several reported periodicities [四] it ranges from hours to hundreds days in HMXBs and from hours to some days in LMXBs. Superorbital periodicities have been detected in both LMXBs and HMXBs. The likely explaination for several systems is the presence of a precessing and tilted accretion disk [2] $]$. Several X-ray transient sources show recurring narrow outbursts separated by long quiescence intervals, explained by the disk instability model [3]. The initial searches for periodicities in the X-ray time series have been involving a few sources at a time. Recently, extensive investigations have been presented by [ [ 4 ], [[]], [ [6] using the RXTE ASM data. Some X-ray binaries observed with Swift BAT have been investigated by [प].

The present work presents the preliminary results of a systematic search for long term periodicities (above one day) of the X-ray binaries in Swift BAT archive, for which to date there are several sporadic detections, but no systematic investigation yet. For comparison, I have searched the periodicities of the same systems in the RXTE ASM data using the same analysis method. The two satellites cover different energy ranges. The higher energies at which BAT is operating is particularly useful to detect periodicities of the highly absorbed sources detected by INTEGRAL IBIS/ISGRI instrument.

\section{Data and analysis}

I have used the daily light curves of the X-ray binaries in the Swift BAT ${ }^{1}$ and RXTE ASM ${ }^{2}$ archives. The Swift satellite is monitoring the sky in the hard X-ray range since 2004. The Burst Alert Telescope (BAT) [8] on board Swift is a coded-mask detector sensitive in the 15-50 keV energy region, routinely used to observe GRBs. A fraction of the sky ranging from $50 \%$ to $80 \%$ is observed each day. During the first ten years of operation, more than nine hundreds sources have been monitored. The Rossi X-Ray Timing Explorer [Q] has been operative from 1996 to 2011. The All Sky Monitor (ASM) used three scanning cameras, each one including a position sensitive proportional counter (PSPC) measuring a shadow pattern of the observed source through a slit. The instrument was sensitive in the range 2-12 keV. Several hundreds sources have been monitored during operation.

Both instruments have secured observations of sources unevenly in time. The spectral analysis has been performed using the SparSpec algorithm [0] to deal with the uneven sampling of the light curves. Data are modelled as the sum of a large number of pure frequencies, choosing the representation with the smallest number of non-zero amplitudes. The algorithm is suitable to deal with multiperiodic time series, often recorded in X-ray binaries where orbital and superorbital periodicities coexist. Depending on the position of the observed object and on the duration of the monitoring, the errors on data points are different, demanding weighting of data. The Swift and RXTE data analyzed in the present paper have been weighted using a modified weighting procedure

\footnotetext{
${ }^{1}$ http://swift.gsfc.nasa.gov/results/transients/

${ }^{2}$ http://xte.mit.edu/ASM_lc.html
} 
suggested by [ए]], [ए2], that takes into account the error on the data and the excess variability of the time series; the procedure is effective for sources spanning a wide range of intensities.

\section{Results}

I present a preliminary overview of the periodicities estimated with the SparSpec algorithm in

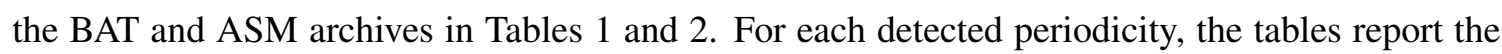
source name, the period (in days) detected in BAT and ASM archives, the type of periodicity, the main references.

\begin{tabular}{|c|c|c|c|c|}
\hline Object & BAT Period $(\mathrm{d})$ & ASM period $(\mathrm{d})$ & Period type & Reference \\
\hline IGR J00370+6122 & 15.7 & 15.7 & Orbital & [四] \\
\hline SMC X-1 & 3.89 & 3.89 & Orbital & [回] \\
\hline $3 \mathrm{~A} 0114+650$ & 11.6 & 11.6 & Orbital & [四] \\
\hline $3 \mathrm{~A} 0114+650$ & 30.7 & 30.7 & Superorbital & [四], [回] \\
\hline LMC X-4 & 30.3 & 30.3 & Superorbital & [四], [焑] \\
\hline 4U 0728-25 & 34.5 & 34.5 & Orbital & [四] \\
\hline Vela X-1 & 8.96 & 8.96 & Orbital & [四] \\
\hline Cen X-3 & 2.09 & 2.09 & Orbital & [四] \\
\hline IGR J11435-6109 & 52.3 & 52.3 & Orbital & [䧃] \\
\hline GX 301-2 & 41.5 & 41.5 & Orbital & [四] \\
\hline H1538-522 & 3.73 & 3.73 & Orbital & [四] \\
\hline IGR J16318-4848 & 79.8 & $\ldots$ & Orbital & [ए5] \\
\hline IGR J16320-4751 & 8.99 & 8.99 & Orbital & []], [回] \\
\hline IGR J16393-4643 & 4.24 & $\ldots$ & Orbital & [四] \\
\hline IGR J16465-4507 & 30.4 & $\ldots$ & Orbital & [ㅁ]], [ए]] \\
\hline IGR J16479-4514 & 3.32 & $\ldots$ & Orbital & [四] \\
\hline IGR J16479-4514 & 11.9 & $\ldots$ & Superorbital & [四] \\
\hline IGR J16493-4348 & 6.78 & $\ldots$ & Orbital & [四], [ए]] \\
\hline EXO 1657-419 & 10.45 & 10.45 & Orbital & [四] \\
\hline 4U 1700-377 & 3.41 & 3.41 & Orbital & [四] \\
\hline IGR J18027-2016 & 4.57 & $\ldots$ & Orbital & [可, [ए5] \\
\hline IGR J18483-0311 & 18.55 & 18.55 & Orbital & [G], [155] \\
\hline XTE J1855-026 & 6.07 & 6.07 & Orbital & [䧃] \\
\hline H1907+097 & 8.4 & 8.4 & Orbital & [䧃] \\
\hline SS 433 & 13.1 & $\ldots$ & Orbital & [䧃] \\
\hline SS 433 & 163 & 163 & Superorbital & [䧃] \\
\hline IGR J19140+0951 & 13.6 & 13.6 & Orbital & [四] \\
\hline EXO 2030+375 & 46 & 46 & Orbital & [四] \\
\hline GRO J2058+42 & 56 & 55 & Orbital & [䧃] \\
\hline SAX J2103.5+4545 & 12.7 & 12.7 & Orbital & [四] \\
\hline 4U 2206+54 & $9.6,19.2$ & 9.6 & Orbital & [四], [可] \\
\hline
\end{tabular}

Table 1: Periodicities of HMXBs: object name, BAT period, ASM period, period type, references

For reference, I report two examples of the spectral analysis, the power spectra of Vela X-1 and Her X-1, in Fig. 四.

The estimated periods in the different energy ranges of BAT and ASM are in agreement. Several orbital periods are detected, confirming the detections by [四] in BAT data and by [四], [目] in 


\begin{tabular}{lllll}
\hline Object & BAT Period (d) & ASM period (d) & Period type & Reference \\
\hline Her X-1 & 34.9 & 34.9 & Superorbital & [䧃], [焑] \\
Rapid Burster & 116,157 & 103,213 & Outburst interval & [䧃], [四] \\
GRS 1747-312 & 138 & 145 & Quasi periodicity & [䧃], [可] \\
\hline
\end{tabular}

Table 2: Periodicities of LMXBs: object name, BAT period, ASM period, period type, references
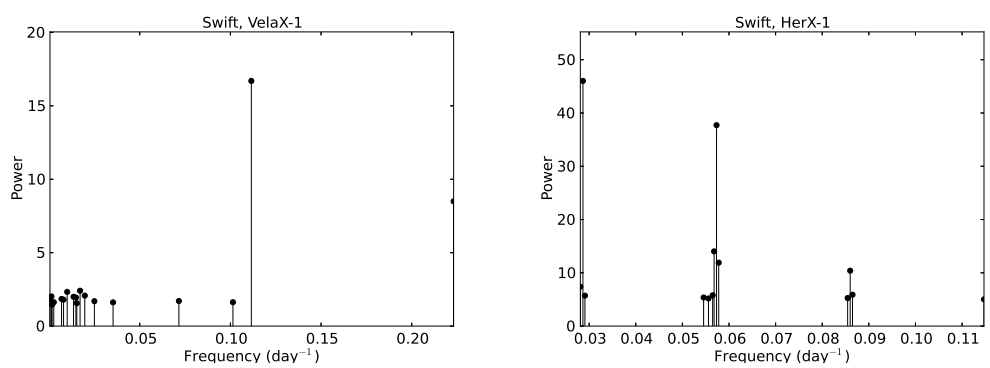

Figure 1: Power spectra of two sources: the HMXB Vela X-1 and the LMXB Her X-1

ASM data. The results for some selected objects of interest for Swift BAT will be briefly discussed in the following.

\section{Discussion}

\subsection{High Mass X-ray binaries}

The SparSpec algorithm detects in Swift BAT data the most part of the periodicities of ASM archive reported by [䧃].

- Vela X-1, a wind accreting X-ray pulsar, shows a clear orbital periodicity (Fig. W)

- The detection of orbital and superorbital periodicities of the supergiant system 3A 0114+650 in both archives confirms the detection by [四] in the BAT data and by [䧃], [四] in the ASM data

- SS 433 is a peculiar source, showing two jets in opposite directions with a $\beta$ factor of 0.26 and precessing with a period of about 164 days [团]; the orbital period is about 13 days; the analysis of the present paper detects both periodicities in BAT data

- During the INTEGRAL scans of the Galactic plane, several highly absorbed HMXB systems have been discovered, after the detection of the first system IGR J16318-4848 [ए13]: IGR J16320-4751 (AX J1631.9-4752), IGR J16393-4641, IGR J16465-4507, IGR J16479-4514, IGR J16493-4348, IGR J 18027-2016 [ए4]]. The physical characteristics of these system are more easily investigated at higher energies. In fact, the orbital period is detected in BAT data for all mentioned systems, and only in one case in ASM data [15], [ए6], [ए7]. The superorbital period of IGR J16479-4514 is also detected in BAT data

- SMC X-1 shows the orbital periods in both data sets, as in [⿴囗⿴囗十⿴囗⿱一一心]; in addition, there is a superorbital quasi periodicity peaked at about 56 days, confirming the suggestion by [四], [目] 
- The system 4U 2206+54 has been previously investigated both in ASM and BAT data. The data of the first years of ASM showed a 9.6 days period [[18], see also [四]. The analysis of BAT record suggested a 19.2 days period, twice the previous value [ए2], as the most recent data [ [] ], [प2], pointing to a secular change of the periodicity, instead of an energy dependent effect. The 9.6 days period is detected in BAT and ASM data, while the 19.2 days period is evident only in BAT data

\subsection{Low Mass X-ray binaries}

As above, the SparSpec algorithm detects in BAT data the most part of the periodicities of ASM archive reported by [四].

- Her X-1 shows a clear superorbital periodicity at about 35 days (Fig. W), confirming the suggestions by [四], [目]

- The Rapid Burster (X1730-333) is a neutron star system in the globular cluster Liller 1. It shows outburst with a variable recurrence time, about 200 days or 100 days. The first period is reported by [四], [目] during the first years of ASM operation; the shorter period coexists with previous one, together with other short periods [6]. The change of the recurrence time from about 200 days to about 100 days at the end of 1999 has been reported by [10]. I confirm the presence of 100 and 200 days periods in ASM data. The BAT data show periodicities at about 100 and 160 days; the new period could be due to an evolution of the outburst recurrence time, see also [[20] who suggests a recurrence time of 160 days in the ASM data from 1996 to 2005

\section{Conclusions}

I have presented the preliminary results of a systematic search for periodicities of the X-ray binaries in the Swift BAT and RXTE ASM observations, to discover new periodicities and to confirm previously reported one. The investigation has detected several orbital and superorbital periodicities in BAT data, confirming the ones detected in ASM data. Generally periodicities are detected in both archives, i. e. both in soft and hard X-rays. I confirm that orbital periodicities are more frequently detected in HMXBs than in LMXBs, as suggested by [四], [2]].

\section{Acknowledgments}

The Swift BAT transient monitor results have been provided by the Swift BAT team. The RXTE ASM results have been provided by the ASM/RXTE teams at MIT and at the RXTE SOF and GOF at NASA's GSFC. The SparSpec software is available at http://www.ast.obs-mip.fr/Softwares

\section{References}

[1] N. E. White et al., The properties of X-ray binaries, in X-Ray Binaries, Cambridge University Press, Cambridge 1995.

[2] J. E. Pringle, Self-induced warping of accretion discs, MNRAS 281 (1996) 357. 
[3] J.-P. Lasota, The disc instability model of dwarf novae and low-mass X-ray binary transients, NewAR 45 (2001) 449.

[4] L. Wen et al., A Systematic Search for Periodicities in RXTE ASM Data, ApJS 163 (2006) 372.

[5] A. M. Levine et al., An Extended and More Sensitive Search for Periodicities in Rossi X-Ray Timing Explorer/All-Sky Monitor X-Ray Light Curves, ApJS 196 (2011) 6.

[6] M. M. Kotze and P. A. Charles, Characterizing X-ray binary long-term variability, MNRAS 420 (2012) 1575.

[7] R. H. D. Corbet and H. A. Krimm, Superorbital Periodic Modulation in Wind-accretion High-mass X-Ray Binaries from Swift Burst Alert Telescope Observations, ApJ 778 (2013) 45.

[8] H. A. Krimm et al., The Swift/BAT Hard X-Ray Transient Monitor, ApJSS 209 (2013) 14.

[9] A. M. Levine at al., First Results from the All-Sky Monitor on the Rossi X-Ray Timing Explorer, ApJL 469 (1996) L33.

[10] S. Bourguignon et al., SparSpec: a new method for fitting multiple sinusoids with irregularly sampled data, A\&A 462 (2007) 379.

[11] R. Corbet et al., Periodicities in X-Ray Binaries from Swift/BAT Observations, PThPS 169 (2007) 200.

[12] R. H. D. Corbet et al., Swift BAT and RXTE Observations of the Peculiar X-Ray Binary 4U 2206+54: Disappearance of the 9.6 Day Modulation, ApJ 655 (2007) 458.

[13] R. Walter et al., INTEGRAL discovery of a bright highly obscured galactic X-ray binary source IGR J16318-4848, A\&A 411 (2003) L427.

[14] R. Walter et al., XMM-Newton and INTEGRAL observations of new absorbed supergiant high-mass $X$-ray binaries, $A \& A 453$ (2006) 133.

[15] C. Jain et al., Orbital X-ray modulation study of three supergiant HMXBs, RAA 9 (2009) 1303.

[16] V. La Parola et al., Detection of an orbital period in the supergiant high-mass X-ray binary IGR J16465-4507 with Swift-BAT, MNRAS 405 (2010) L66.

[17] G. Cusumano et al., Hard X-ray monitoring of HMXB with Swift-BAT: a systematic search to reveal long term periodicities, in Proceedings of the 8th INTEGRAL Workshop "The Restless Gamma-ray Universe" (INTEGRAL 2010), PoS(INTEGRAL2010) 132.

[18] R. H. D. Corbet and A. G. Peele, 4U 2206+54: An Unusual High-Mass X-Ray Binary with a 9.6 Day Orbital Period But No Strong Pulsations, ApJ 562 (2001) 936.

[19] N. Masetti, A change in the outburst recurrence time of the Rapid Burster, A\&A 381 (2002) L45.

[20] V. Šimon, Long-term activity of the Rapid Burster, IAUS 230 (2006) 47.

[21] P. C. Joss ans S. Rappaport, Highly compact binary X-ray sources, A\&A 71 (1979) 217. 